\title{
Developing time to frequency-domain descriptors for relaxation processes: Local trends
}

\author{
Juan S. Medina ${ }^{\mathrm{a}, *}$, Daniel J. Arismendi-Arrieta ${ }^{\mathrm{a}}$, Jóse V. Alemán ${ }^{\mathrm{b}}$, Rita Prosmiti ${ }^{\mathrm{a}, * *}$ \\ ${ }^{a}$ Institute of Fundamental Physics, CSIC (IFF-CSIC), Serrano 123, 28006 Madrid, Spain \\ ${ }^{b}$ Departamento de Química, Facultad de Ciencias del Mar, ULPGC, Campus Universitario de Tafira, 35017 Las Palmas de G. \\ Canaria, Spain
}

\begin{abstract}
It is common practice while studying complex liquids to analyze their relaxations in time as well as in frequency. Unfortunately, there are not often at hand short and compact expressions corresponding simultaneously to the mathematical formulation of a same phenomenon in both spaces. Therefore, this work is focused towards the approximation of Fourier Transform of certain Weibull distributions (the time derivative of the Kohlrausch-Williams-Watts function) by Havriliak-Negami functions. In particular, it was found that a small interval of low frequencies are needed to recover the main traits of the relaxation for the stretched $(\beta \leq 1)$ and squeezed $(\beta>1)$ instances. However, it's easily recognizable that the weight of the low frequency part competes with the weight of the high frequency part, and the former distorts the power law behavior, diverging from $-\beta$. In consequence, the tail's sturdiness influences the asymptotic trend of HN, suggesting a careful design of the approximant, the method of optimization, the absent of data errors, and of course the frequency domain. In this sense, we were able to explain how the asymptotic laws naturally emerge as a function $\omega$, and validate the suitability-flexibility-instability of our local approximants.
\end{abstract}

Keywords: relaxation functions, simple/complex liquids, frequency and time domains

\section{Introduction}

Many relaxation phenomena in simple or complex fluids are most often fitted as a function of time by the function of Kohlrausch-Williams-Watts [1, $2,3,4,5]$, or as a function of frequency such as Debye [6], Cole-Cole [7], Cole-Davidson [8], Havriliak-Negami [9, 10]. The Kohlrausch relaxation function, have become ubiquitous in many areas of Physics and Chemistry, from the discharge of capacitors and dielectric properties of polymers, to the study of complex systems and autocorrelation functions in molecular dynamics $[11,12,13,14,15$, $16,17,18,19,20,21,22,23,24,25,26]$, as well

\footnotetext{
${ }^{*}$ Corresponding author. Tel:+34 915616800 ext 941143

** Corresponding author. Tel:+34 915616800 ext 941131

Email addresses: tlazcala@yahoo.es (Juan S.

Medina), rita@iff.csic.es (Rita Prosmiti)
}

as in soft-matter [27, 28, 29, 30, 31, 32, 33, 34, 35]. In such cases, any ensemble of interacting elements organized in multiscale clusters, whose local relaxations, or restructuring bonds jump with random delay times of type $t^{(-1-\beta)}$, should present an autocorrelation, or decay, of Kohlrausch's nature [28, 36, $37,38]$. Despite of its simple form, the relation between the $\beta$ parameter and the thermodynamic conditions (temperature, pressure, etc) at which a relaxation process occurs lack of a direct connection and interpretation, making a very difficult task for the experimentalists and theoreticians to disentangle its hierarchical structure.

Such facts, advises us to study relaxation behavior not only through its dynamical response along time but also in other spaces of representation, such as of frequency [39, 40, 19, 34, 41, 42, 43, 44, 45]. However, there is no obvious mathematical approach for 
an analytical and compact transformation from time to frequency domain. Besides the existence of its Fourier and Laplace transforms for $0<\beta \leq 2$, it also presents several problems of convergence which is possible to get round with numerical methods or resummation of series $[46,47,48,49,50,16]$. Nevertheless, a concise mathematical formula to give account, even approximately, of such transforms would make it easier to compare with the most common mathematical functions in the complex domain. Additionally, it would be of great utility and will provide a valuable set of techniques for employing in different analytical and laboratory procedures. For example, to accelerate the calculations or evaluate repeatedly such functions; in analysis and filtering of data by identifying the existence of superposed signals, or removing strong noise [48, 49]; as well as to provide an exhaustive account of characteristic relaxation times -real or virtual-[19, 34, 41], and justify the underlying dominion behavior in diverse mechanisms [40, 19, 34, 41, 42, 44, 45, 51, 52].

In this sense, a considerable effort to provide a theoretical background and interconnect both spaces have been reported in the literature. However, such representations relies on numerical assumptions or non-closed analytical representations. So far, most of the studies have focused on the stretched exponential case $(0<\beta \leq 1)$, and very little is known about the squeezed or compressed case $(1<\beta \leq 2)$. Hence, we examined here, issues related with the asymptotic behavior of the Kohlrausch's (or Weibull's [53, 54]) FT in the frequency domain, its possible description through an analytical form, and the type of function or combination of them to represent the original data in the whole range of frequencies. These points will be addressed in the following, by showing how a series of approximations provides a good description in both low and high frequencies, and further, how each term shares its contribution to the local structure of a Kohlrausch relaxation function.

The article is organized as follows: In Sec. 2 we give the analytical and computational considerations employed in the study, while in Sec. 3 we present the results by decomposing the shape parameter $\beta$ in two, $\beta \leq 1$ and $\beta>1$, intervals. In each case, we examine the asymptotic behavior by numerical samplings in $\omega$-space and extending the limits in $t$-space. The description of high frequencies decays is given by a unique set of strict $\mathrm{HN}$ functions when $\beta \leq 1$, and with a set of the same kind, although parametrically extended if $\beta>1$. The discussion of our results, and their comparison with previous studies are presented in Sec. 4, while in Sec. 5 we give some conclusions and further considerations.

\section{Analytical and computational considerations}

We introduce the notation, $\phi_{K, \beta}(t) \equiv \exp -t^{\beta}$ for the Kohlrausch relaxation function, $0 \leq t<\infty, \beta \leq 1$, and notice that we use here dimensionless variables solely (normalized), [19] for both times and frequencies, i.e. $t / \tau_{K} \mapsto t$ and $\omega \tau_{K} \mapsto \omega$. Then, for the one-sided FT $\chi_{\beta}(\omega)=\int_{0}^{\infty} e^{-i \omega t} \phi_{K, \beta}(t) \mathrm{d} t$ and for minus the transformation of the Weibull distribution we have $\psi_{\beta}(\omega)=-\int_{0}^{\infty} e^{-i \omega t} \frac{d}{d t} \phi_{K, \beta}(t) \mathrm{d} t$, both related in $\omega$-space by $\psi_{\beta}(\omega)+i \omega \chi_{\beta}(\omega)=1$. The modulus of function $\psi_{\beta}(\omega)$ presents the following asymptotic behavior in the domain of frequencies: $\left|\psi_{\beta}(\omega)\right| \sim 1$ when $\omega \rightarrow 0$ and $\left|\psi_{\beta}(\omega)\right| \sim \Gamma(\beta+1) / \omega^{\beta}$ as $\omega \rightarrow$ $\infty$, being monotonically decreasing in the $\omega$-values and strongly depending on the value of the parameter $\beta$. Therefore, we will show when approximating the mentioned transform, how the parameters of the HN function [9, 10], $H N_{\alpha, \gamma, \tau, \lambda}(\omega)=\frac{1}{\left(1+\left(i \omega \tau_{H N}\right)^{\alpha}\right)^{\gamma}}$, $0<\alpha, \gamma \leq 1$, are uniquely determined by the parameter $\beta$.

In short we have then the $\mathcal{A} p_{1} H N$ and $\mathcal{A} p_{2} H N$ approximants:

$$
\begin{aligned}
& \mathcal{A} p_{1} H N=\psi_{\beta}(\omega) \approx \frac{\lambda}{\left(1+(i \tau \omega)^{\alpha}\right)^{\gamma}} \\
& \mathcal{A} p_{2} H N=\psi_{\beta}(\omega) \approx \sum_{s=1}^{2} \frac{\lambda_{s}}{\left(1+\left(i \tau_{s} \omega\right)^{\alpha_{s}}\right)^{\gamma_{s}}}
\end{aligned}
$$

with share coefficients $\lambda$ and $\lambda_{1} \equiv \lambda$ and $\lambda_{2}=$ $1-\lambda_{1}$ in Eqs. 1 and 2, respectively. It was shown that, a double approximant of $\mathrm{HN}$ functions describe fairly well the FFT of the Weibull distribution, as well as the Cole-Davidson-Kohlrausch family $[19,34]$. The question is then how sensitive are the parameters obtained during the optimization to reproduce the asymptotic laws indexed to them (e.g. $\alpha_{i} \cdot \gamma_{i}=\alpha_{i} \cdot \gamma_{i}(\beta)$ when the rest are also functions of $\beta)[41,42,55]$. 
The range of simulated $\beta$ parameters corresponds to the stretched instance with $0<\beta \leq 1$ and the squeezed or compressed instance for $1<\beta \leq 2$. The chosen grid points have a variable size step of 0.1 with intermediate values of 0.03 in the whole range of $\beta$ and a starting point of 0.02 . To this end we have used two domains of frequencies. One of them comprehends the range of $v=0-500$ (low to medium) being $\omega=2 \pi v$, as it was given in Ref. [19], while the other one extends to higher frequencies with $v=0-10^{12}$ if $\beta \leq 1$ and $v=0-10^{7}$ for $\beta \geq 1$. The reason for these distinct intervals is due to the increasing numerical noise that overshadows the signal, while the large domain is to provide a concise and compact mathematical description. That could be helpful in exploring different kind of relaxations, associated to several size scales and diverse phenomena, as well as to reconstruct signals with high accuracy, based on rigorous criteria instead of arbitrary ones.

Finally, there will be three general sampling steps $\left(\mathrm{r}_{1}, \mathrm{r}_{2}\right.$ and $\left.\mathrm{r}_{s l}\right)$ and three particular ones $\left(\mathrm{r}_{2 b}, \mathrm{r}_{2} *\right.$ and $\left.\mathrm{r}_{s l}{ }^{*}\right)$, which can be seen as implicit weights during the optimization procedure. The $r_{1}$ and $r_{2}$ are associated with a narrow frequency domain (0-500), while $\mathrm{r}_{s l}$ is associated to a wider domain, $0-10^{7}(\beta \geq 1)$ and $0-10^{12}(\beta \leq 1)$, respectively. The $\mathrm{r}_{1}(\delta v=0.5)$ is a linear coarse mesh dominated by tail values in medium frequencies, with a residual influence of low frequencies. The $\mathrm{r}_{2}(\delta v=0.001)$, is a linear fine mesh with many points mainly contributing to the low frequencies, while, $\mathrm{r}_{s l}$ is a logarithmic homogeneous mesh with a thousand points for each decade of frequency, weighting less the medium and low frequencies than $r_{1}$ due to the logarithmic scale. In other words, $\mathrm{r}_{1}$ and $\mathrm{r}_{s l}$ are both pruned of low frequencies, weighting mostly medium to high frequencies, thus the corresponding result will properly describe the asymptotic tail behavior, while the $\mathrm{r}_{2}$ and $\mathrm{r}_{s l}$ are our standard grids to differentiate between low and high frequency features, while the $\mathrm{r}_{2 b}, \mathrm{r}_{2} *$ and $\mathrm{r}_{s l} *$ cases will be described later on.

To assess the influence of a finite-size frequency window over $\beta$, for each of the sampling meshes mentioned before, we generated the corresponding reference data of the FT for $\psi_{\beta}(\omega)$ employing the Mathematica $^{\mathrm{TM}}$ [56] package. We choose this pack- age due to its accuracy and for avoiding numerical oscillations as far as possible. In turn, we employed the graphical interface for non-linear fitting of xmgrace [57], with the intention of designing the best possible approximation and test how the HN function, expressed as the approximants of Eq. 1 and Eq. 2 sticks to the data.

\section{Results}

In this section we present our results related with an adjustment of a probe function, such as, its type, its flexibility to describe the reference data in the whole range (low and high) of frequencies, the sensibility of its parameters to finite-size effects coming from a coarse or fine mesh of sampling, and then a short compact relationship between the parameters in $\omega$-space and $t$-space.

\subsection{The stretched instance $\beta \leq 1$}

\subsubsection{Asymptotes of the reference data}

In Figure 1 (see upper and lower left-side panels) we show the first and second logarithmic derivatives of $\left|\psi_{\beta}\right|$ as a function of $v$ and $\beta$ for the $r_{s l}$ sampling mesh. One can see the evolution of $\left(\log _{10}\left|\psi_{\beta}\right|\right)^{\prime}$ identifying three regions corresponding to the plateau, bend-fall and tail, respectively. The plateau appears at low frequencies and then drops down (bend-fall) in the neighborhood of $v \sim 10^{-1}$, reaching a constant tail after $v \approx 20$ and approaching to its horizontal asymptote value of $\beta$. In the lower panel we observe smooths peaks for the $\left(\log _{10}\left|\psi_{\beta}\right|\right)^{\prime \prime}$, almost symmetrical, and of contracting half-width, which maximum moves from $v_{\text {max }} \approx 10^{-2}$ to $v_{\text {max }} \approx 2 \times 10^{-1}$ as $\beta$ goes from 0.3 to 1 .

In Figure 1 (top-left panel) one can see that the curves for $\left(\log _{10}\left|\psi_{\beta}\right|\right)^{\prime}$ follows a power decaying law after $v \approx>20$, so by discarding the data corresponding to the plateau-bend-fall, we then proceeded to adjust them to the generic function $M v^{-d}$, with $d \approx \beta$. In the right-side panel of Figure 1, we quantified the importance of low and high frequencies on describing the tails $\left(\mathrm{r}_{2} *\right.$ and $\mathrm{r}_{s l} *$ sampling are obtained from the general $r_{2}$ and $r_{s l}$ samplings for $v>20$ ). It is shown that both series of exponents, $d_{r_{2}} *$ and $d_{r_{s l}} *$, follow closely the line corresponding to an ideal exponent $\beta$. In the $\mathrm{r}_{2} *$ case, it shows a slight bulge in 

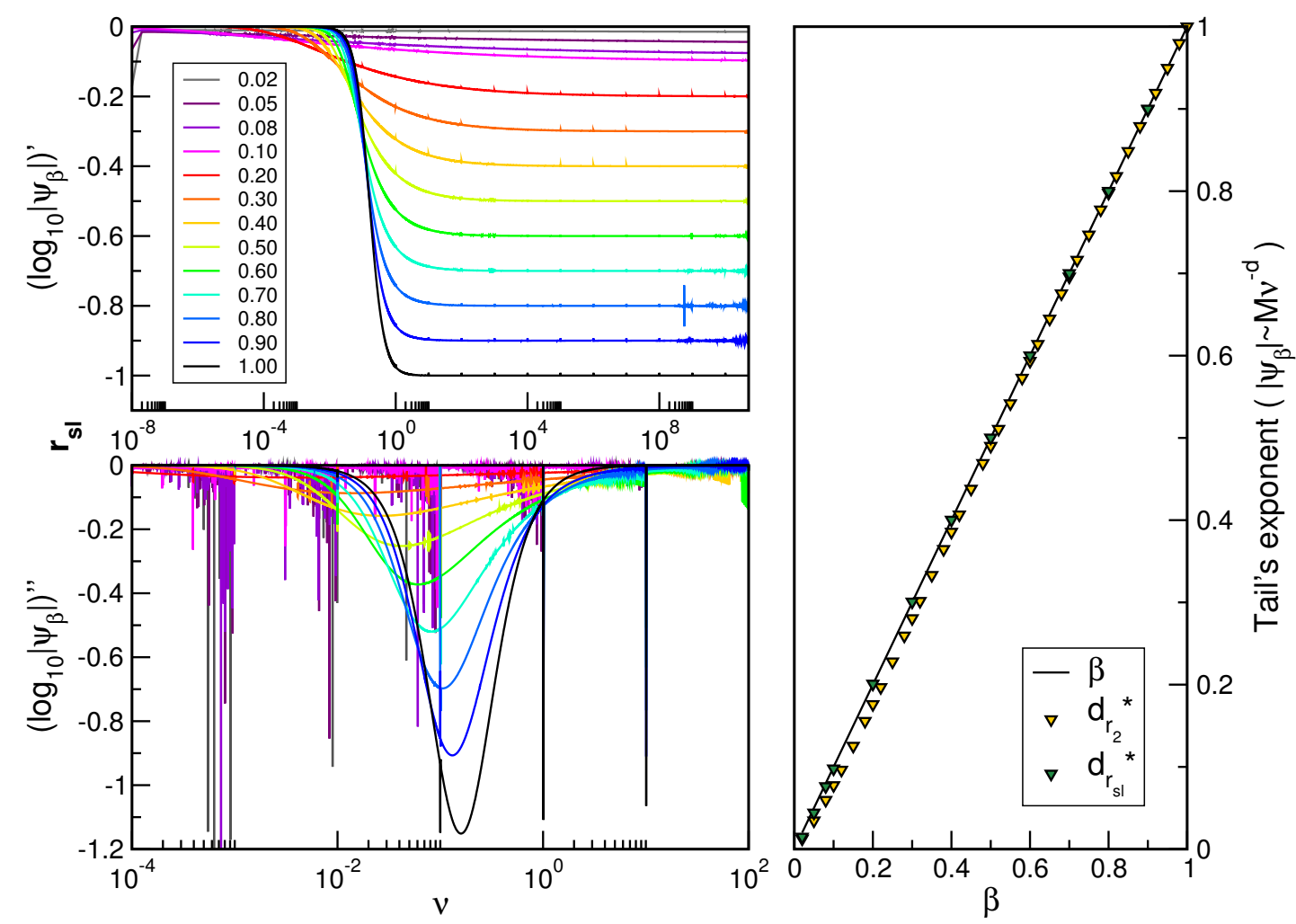

Figure 1. Left side (upper and lower panels), $1^{\text {st }}$ and $2^{\text {nd }} \operatorname{logarithmic}$ derivatives of $\log _{10}\left|\psi_{\beta}(\omega)\right|$ for the $\mathrm{r}_{s l}$ sampling mesh, as a function of $v$ and $\beta$ in the interval of $0<\beta \leq 1$. Right side, comparison between the tail exponent $d$ (triangles down) $v s \beta$ for the $\mathrm{r}_{2} *$ and $\mathrm{r}_{s l} *$ sampling meshes.

the interval $0.10 \leq \beta \leq 0.30$ where the absolute error is greater, although the relative error $\left(\beta-d_{r_{2}^{*}}\right) / \beta$ decreases in the path from $\beta=0.02$ to $\beta=1$. This is a consequence of the slower relaxation of the logarithmic slope, $\left(\log _{10}\left|\psi_{\beta}\right|\right)^{\prime}$, towards its asymptote for small $\beta$. The $\mathrm{r}_{s l} *$ case corroborates this, with relative errors even smaller than the previous case for each $\beta$.

\subsubsection{Trends of $\mathcal{A} p_{1} H N_{\alpha, \gamma, \tau, \lambda}(\omega)$}

In Figure 2 we display the sensibility of the parameters $\alpha$ and $\gamma$ using the $\mathcal{A} p_{1} H N_{\alpha, \gamma, \tau, \lambda}(\omega)$ approximant as a function of $\beta$ for all the three $\mathrm{r}_{1}, \mathrm{r}_{2}$ and $\mathrm{r}_{2 b}$ samplings. Notice that in the case of $\mathrm{r}_{2 b}$, the initial condition $H N_{\alpha, \gamma}(\omega=0)=1$ is relaxed to a free value, i.e. $\psi_{\beta}(\omega) \approx \lambda * H N_{\alpha, \gamma}(\omega)$ with $\lambda$ to be determined in the optimization. The idea behind this is to allow the HN approximant compensates its lack of suitability in the very low frequencies, and to soften the influence of the tail in relationship to the body (plateau-bend-fall) part of data. In turn, the $\alpha_{r_{1}}(\beta)$ and $\gamma_{r_{1}}(\beta)$ shows a monotonous increasing behavior and the curves cross at $\beta=1$. In the case of $\alpha_{r_{2}}(\beta)$ and $\gamma_{r_{2}}(\beta)$ the curves

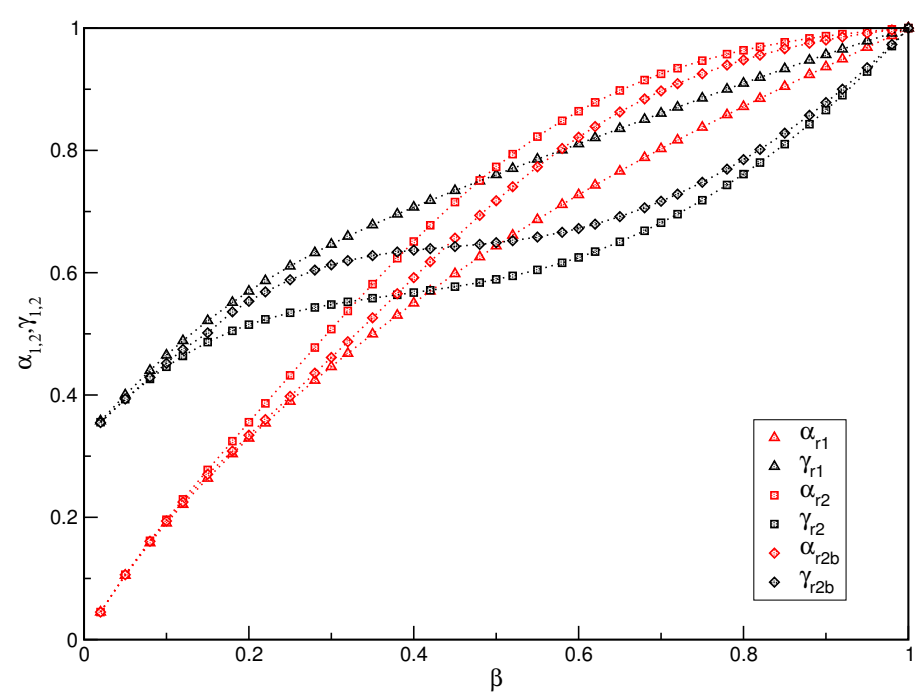

Figure 2. Parameters $\alpha$ and $\gamma$ obtained from adjustment to the $\mathcal{A} p_{1} H N$ approximant in the interval of $0<\beta \leq 1$. The coarser sampling step is for $r_{1}$ mesh (triangles up). Finer steps are those of $r_{2}$ (squares) and $r_{2 b}$ (diamonds). For $\mathrm{r}_{1}$ and $\mathrm{r}_{2} \lambda \equiv 1$, while $\mathrm{r}_{2 b} \lambda \neq 1$. Dotted lines are just guides to the eyes. 
shows the same behavior with an additional crossing (approx. at $0.32<\beta<0.35$ ). In both cases the value of $\lambda$ is 1 . Finally, the $\alpha_{r_{2} b}(\beta)$ and $\gamma_{r_{2} b}(\beta)$ curves are lying between $r_{1}$ and $r_{2}$ with the crossing shifted to the right of the former $(0.42<\beta<0.45)$, while $\lambda$ presents small variations around 1 , with maximum of a $6 \%$ around $\beta=0.32$. A crossing between $\alpha$ and $\gamma$ curves might indicate how the implicit weights shapes the transition between the dominant parts of the curves, and how the approximant function should adjust itself to the body, tail, or both. We have then an enhancement of the weight of tail in detriment of body weight from $r_{2}$ to $r_{1}$, while for the $r_{2 b}$ the constraint of approximating the low frequency zone by the correspondent region of $\mathrm{HN}$ function allows a greater influence on the remaining part by the data of high frequency.

\subsubsection{Trends of $\mathcal{A} p_{2} H N_{\alpha, \gamma, \tau, \lambda}(\omega)$}

In this section we discuss the contribution of each $H N_{s=1,2}(\omega)$ to the local structure of the Kohlrausch function. In Figure 3 are presented the fitting parameters for the $r_{1}$ (upper panels), $r_{s l}$ (medium panels) and $r_{2}$ (lower panels) sampling meshes as a function of $\beta$. In the right panels we plot the $\alpha_{1,2}$ and $\gamma_{1,2}$ parameters, responsible for the power law behavior of tails, while the left panels show the characteristic times of both functions, $\tau_{1,2}$, and share coefficient $\lambda$ which measures the weight balance between both functions. On one hand, in the case of $r_{1}$, we should notice the slowing varying $\lambda$ in the interval $0.02 \leq \beta \lesssim 0.6$ with a value close to 0.5 with both $H N_{s=1,2}$ equally contributing. Then it suddenly drops to 0 from $\beta \approx 0.60$ to 1 . Also, it is remarkable the difference in magnitude between $\tau_{1}$ and $\tau_{2}$. While $\tau_{2}$ never exceeds the value of $1.1, \tau_{1}$ requires of a decimal logarithmic scale to be shown in the same graph. This indicates that at low frequencies the dominant function $\mathrm{HN}_{2}$ is only corrected by the association with $H N_{1}$, an oversimplified picture because it is more accurate when $\beta \rightarrow 1$, than $\beta \rightarrow 0$. On the other hand, $\alpha_{2}$ and $\gamma_{2}$ do not cross. Additionally, there is an apparent discontinuity at $\beta=1$ for $\alpha_{1}$ and $\gamma_{1}$ due to the fact that $\lambda$ drops down to zero. This however, might be seen as $H N_{1}(\omega)$ serves as a backup to $H_{2}(\omega)$ while fitting the functions in the low frequency zone, and that one of the $H N(\omega)$

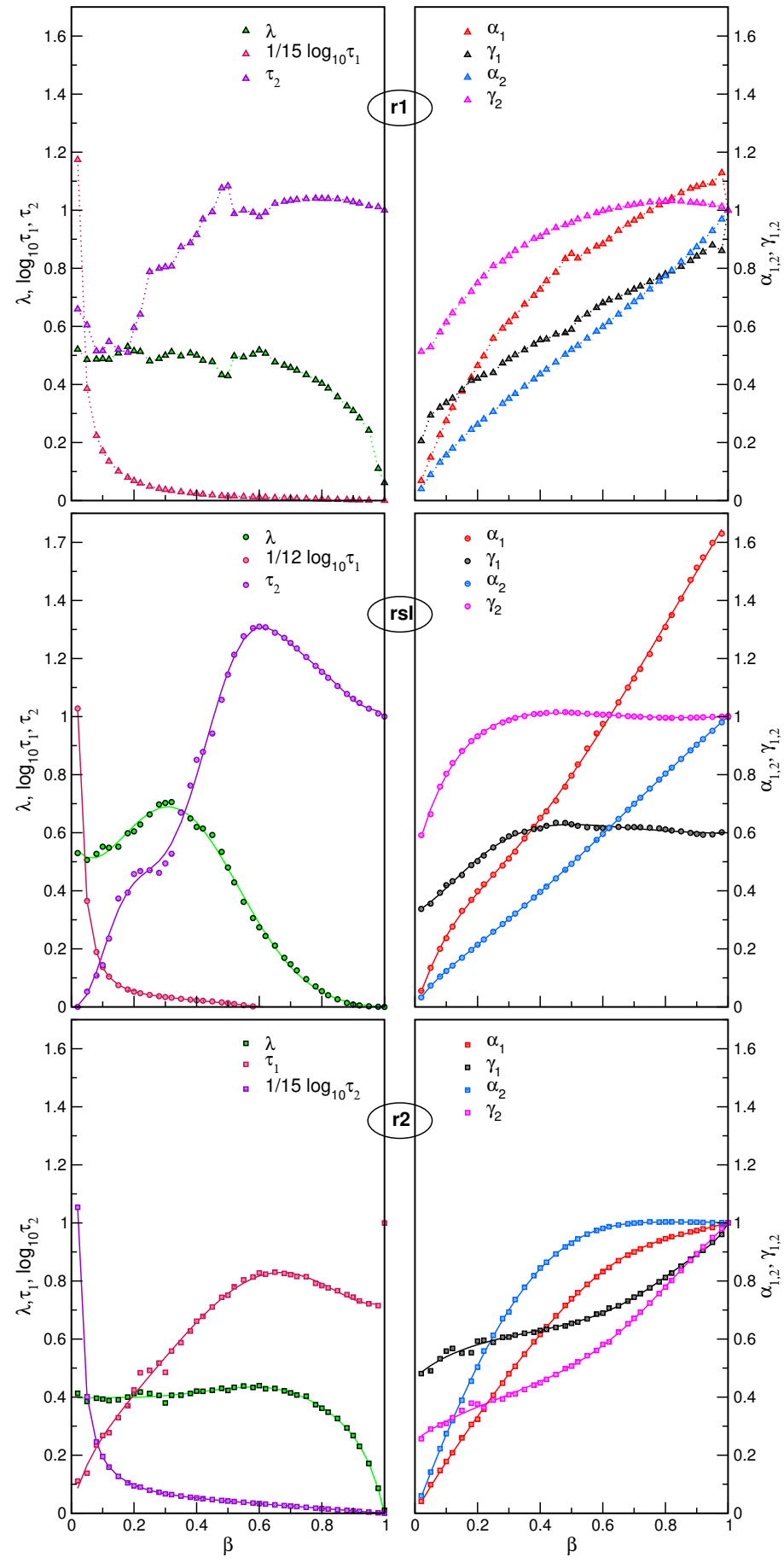

Figure 3. Parameters obtained from adjustment to the $\mathcal{A} p_{2} H N$ approximant in the interval of $0<\beta \leq 1$. On the left side are $\tau_{1,2}$ and $\lambda$, while at the right side are $\alpha_{1,2}$ and $\gamma_{1,2}$. Subscripts 1 and 2 are for the $1^{\text {st }}$ and $2^{\text {nd }} \mathrm{HN}$ terms (see Eq. 2). Triangles up corresponds to the $r_{1}$ sampling mesh (upper panels), while circles and squares corresponds to $r_{s l}$ (medium panels) and $r_{2}$ (lower panels), respectively. Dotted lines are just guides to the eyes for $r_{1}$ grid, while solid lines corresponds to mathematical expressions (See Tables 1-4 in appendix) for the grids $\mathrm{r}_{2}$ and $\mathrm{r}_{s l}$, respectively. 
cannot contribute anymore during the optimization.

In the case of $r_{s l}$, we observe important differences with respect to the previous case. The share coefficient $\lambda$ do not seem steady as before, indicating the loss of weight between both $H N_{s=1,2}$, with $\tau_{s}$ curves having a smoother behavior. One should notice that the opposite result found of $\tau_{1}$ and $\alpha_{1}$, implies a very quick decay of $H N_{1}(\omega)$, while a slow down of such decay when the value of $\alpha_{1}$ is small. This suggests a strong source of error, possibly an interference between $H N_{1}(\omega)$ and $H N_{2}(\omega)$ in the initial interval $0.02 \leq \beta<0.18$, not only for this sampling step but also for $r_{1}$. This balance allows a slight rivalry with the values of $H N_{2}(\omega)$ also dominated by a small $\alpha_{2}$. We should add that interferences in the vicinity of $\beta \sim 1$ between $H N_{1}(\omega)$ and $H N_{2}(\omega)$ also occur affecting $\tau_{1}$, as this curve shows a discontinuity at $\beta=1$, since $\tau_{1}(\beta=1)=1$ and $\tau_{1}(\beta \lesssim 1)<1$. One can see that $\alpha_{1}$ and $\gamma_{1}$ also take values quite far from 1 when $\beta \lesssim 1$; which at the end is partially consequence of a negligible $\lambda$ in that zone, pointing to a minor and complementary role of $H N_{1}(\omega)$ describing $\psi_{\beta \lesssim 1}(\omega)$ tail. This trend is then opposite when the $\beta$ values are smaller than $\beta \sim 0.4$, which entails the fact that tails are not fully developed at very high frequencies for values of $\beta<0.4$. We also should point out that the quasi constant behavior of $\gamma_{1,2}$ in the interval $0.40 \lesssim \beta<1$, and the quasilinear one of $\alpha_{1,2}$ in the whole interval of $\beta$, suggest that the relaxation of tails for $\mathrm{HN}_{2}(\omega)$ are nearer to a Cole-Cole type, $(\gamma \equiv 1)$, than to a Cole-Davidson one, $(\alpha \equiv 1)$. By contrast, relaxation for $H N_{1}(\omega)$ still remains $\mathrm{HN}$ type in both $r_{1}$ and $r_{s l}$ cases though.

In turn, in the case of $r_{2}$, we found that the roles of characteristics times $\tau_{1}$ and $\tau_{2}$ are interchanged. Now $\tau_{1}$ scale does not surpass the value of 1 , suggesting two scenarios in the whole range of $\beta$. One in which the first term of the approximant, $H N_{1}(\omega)$, losses progressively weight as $\beta$ increases (starts at $\beta \approx 0.62$ ), explaining the discontinuity of $\tau_{1}$ at $\beta=1$. On the contrary, in the second scenario $(0.02 \leq \beta<$ $0.62) \lambda \approx 0.4$ and the difference in contribution between both terms is due to the very distinct scale of time for $\tau_{1}$ and $\tau_{2}$. This situation allows to the approximant $\mathcal{A} p_{2} H N_{\alpha, \gamma, \tau, \lambda}(\omega)$ a description for a wide range of frequencies without excessive gap with data, $\psi_{\beta}(\omega)$. Pairs $\alpha_{1}, \gamma_{1}$ and $\alpha_{2}, \gamma_{2}$ show a similar behavior between them, crossing themselves and with shapes as in the case of $\mathcal{A} p_{1} H N_{\alpha, \gamma, \tau, \lambda}$ (see Figure 3).

In order to provide a relationship between the HN parameters of the $\mathcal{A} p_{2} H N$ approximant and $\beta$, and aiming to interconnect time and frequency domain in a compact and analytical form, further preliminary mathematical relationships were obtained from adjustment to the data show in Figure 3. Such expressions (see Appendix A) could be helpful for further model refinements, as well as for the adjustment of any set of real data, e.g. experimental ones.

\subsection{The squeezed or compressed instance $\beta>1$}

\subsubsection{Asymptotes of the reference data}

As in the stretched case, in Figure 4, the $\left(\log _{10}\left|\psi_{\beta}\right|\right)^{\prime}$ and $\left(\log _{10}\left|\psi_{\beta}\right|\right)^{\prime \prime}$ as a function of $v$ and $\beta$ for the $r_{s l}$ sampling mesh (left side upper and lower panels) are represented. When compared to the stretched case there is a qualitatively different response, with an abrupt change of direction between the body (plateau-bend-fall-well-raise) at medium frequencies and the high frequency tail. The first logarithmic derivative it is no longer a simple step, and after the fall a narrow well is formed (at $v \lesssim 0.8$ ), which then raises and stabilizes at the selected value of $-\beta$. As a direct consequence, the $\left(\log _{10}\left|\psi_{\beta}\right|\right)^{\prime \prime}$ presents two peaks. The first corresponds to the fall of the body of the $\log _{10}\left|\psi_{\beta}\right|$ function, while the second peak has a positive intensity and is smaller in magnitude than the preceding. It indicates a fold upwards in the curve $\log _{10}\left|\psi_{\beta}\right|$ in a very narrow region of frequencies as the short half-width of the peaks is developed for $v \sim 2$ as $\beta \rightarrow 2$. Beyond this range of frequencies (i.e. $v \geq 10$ ) the "activity" of the second derivative, $\left(\log _{10}\left|\psi_{\beta}\right|\right)^{\prime \prime}$, is negligible and the function is mostly of a potential nature.

Again, as in the stretched instance, in the right side of Figure 4 we show the tail's exponent $d v s$. $\beta$. As before, $d_{r_{2}^{*}}$ corresponds to the adjustment of $M v^{-d}$ to $\log _{10}\left|\psi_{\beta}\right|$, in the interval $v=20-500$ and $d_{r_{s l}^{*}}$ for the fit in the range of $v=20-10^{7}$. It is quite difficult to distinguish the trend of these two sets of parameters from the result $d=\beta$, pointing to an early high frequency tail very different in behavior from the main body of the spectrum. 

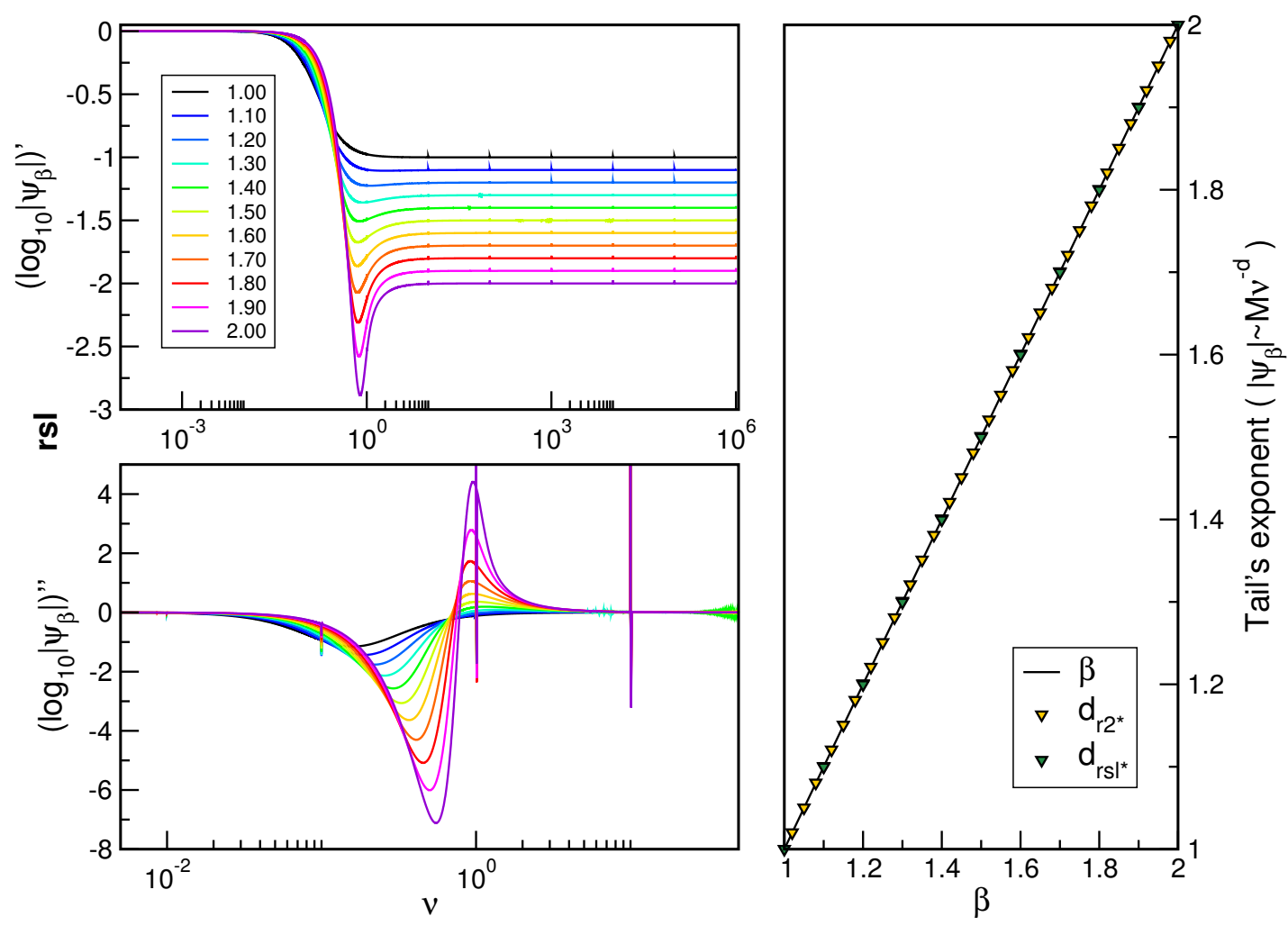

Figure 4. Same as Figure 1, in the interval of $1<\beta \leq 2$. For the sake of clarity not all the $\beta$ values are depicted.

\subsubsection{Trends of both $\mathcal{A} p_{1,2} H N_{\alpha, \gamma, \tau, \lambda}(\omega)$}

In order to show how the local structure of the Kohlrausch function varies when approximated to $\mathcal{A} p_{1} H N$ and $\mathcal{A} p_{2} H N$ for the interval $1<\beta \leq 2$, we should emphasize that the ripples of $|\psi(\omega)|$ around $v \approx 1$ impose large difficulties to follow the data from low to high frequencies. Therefore, the adjustment to $\mathcal{A} p_{1} H N$ or $\mathcal{A} p_{2} H N$ approximants won't work, as the HN function does not show such an extreme changes in curvature. Nevertheless, we conserve the functional form of $\mathrm{HN}$ as support for the cases of $\alpha, \gamma>1$. In Figure 5, we plot the evolution of the fitting parameters $\alpha_{s}, \gamma_{s}$ (right side) and $\lambda_{s}, \tau_{s}$ (left side) for the $r_{2}$ (upper panel) and $r_{s l}$ (lower panel) sampling meshes, with the approximant $\mathcal{A} p_{2} H N$.

In the case of $r_{2}$ (upper panels), the $\gamma_{s}$ parameters obtained after optimization follow a potential law, with no crossing between $\alpha$ and $\gamma$ parameters. We should also point out an almost constant behavior of $\alpha_{1,2} \approx 1$ in the whole interval of $\beta$. Now the magnitudes of the characteristic times $\tau_{s}$ are very similar in the scale, and the balance in weight between both $H N_{s=1,2}$ is preserved for intermediate vales of $\beta$ as it goes from 1 to 2 . In the case of $r_{s l}$ (lower panels), the characteristic times $\tau_{s}$ have a very similar magnitude in the scale, although the share coefficient $\lambda$ is not steady any more. The triplet $\left\{\alpha_{2}, \gamma_{2}, \tau_{2}\right\}$ is describable by means of smooth functions with $\alpha_{2}$ and $\gamma_{2}$ having a quasilinear behavior. Nonetheless this is not the case for the triplet $\left\{\alpha_{1}, \gamma_{1}, \tau_{1}\right\}$, where the last two curves are now broken, with no defined trend. Thus only a piecewise description is admissible for them. Despite the poorly defined trends of $\alpha$ and $\gamma$ type parameters, a finer classification of high frequency spectral data $\left(\mathrm{r}_{s l}\right)$ can be obtained, such as: $\alpha_{1,2} \sim \beta, \gamma_{1} \sim 2$ and $\gamma_{2} \sim 1$, while for the low frequency data $\left(\mathrm{r}_{2}\right)$ will come later on when we discuss the asymptotic laws in the next section.

\section{Discussion and conclusions}

Above, we have examined the deformation of the parameter space as a function of the weights, so here we discuss how the asymptotic laws naturally emerge and explain the deviations when the $\mathcal{A} p_{1} H N$ and $\mathcal{A} p_{2} H N$ approximants are used to adjust the Weibull distribution. 


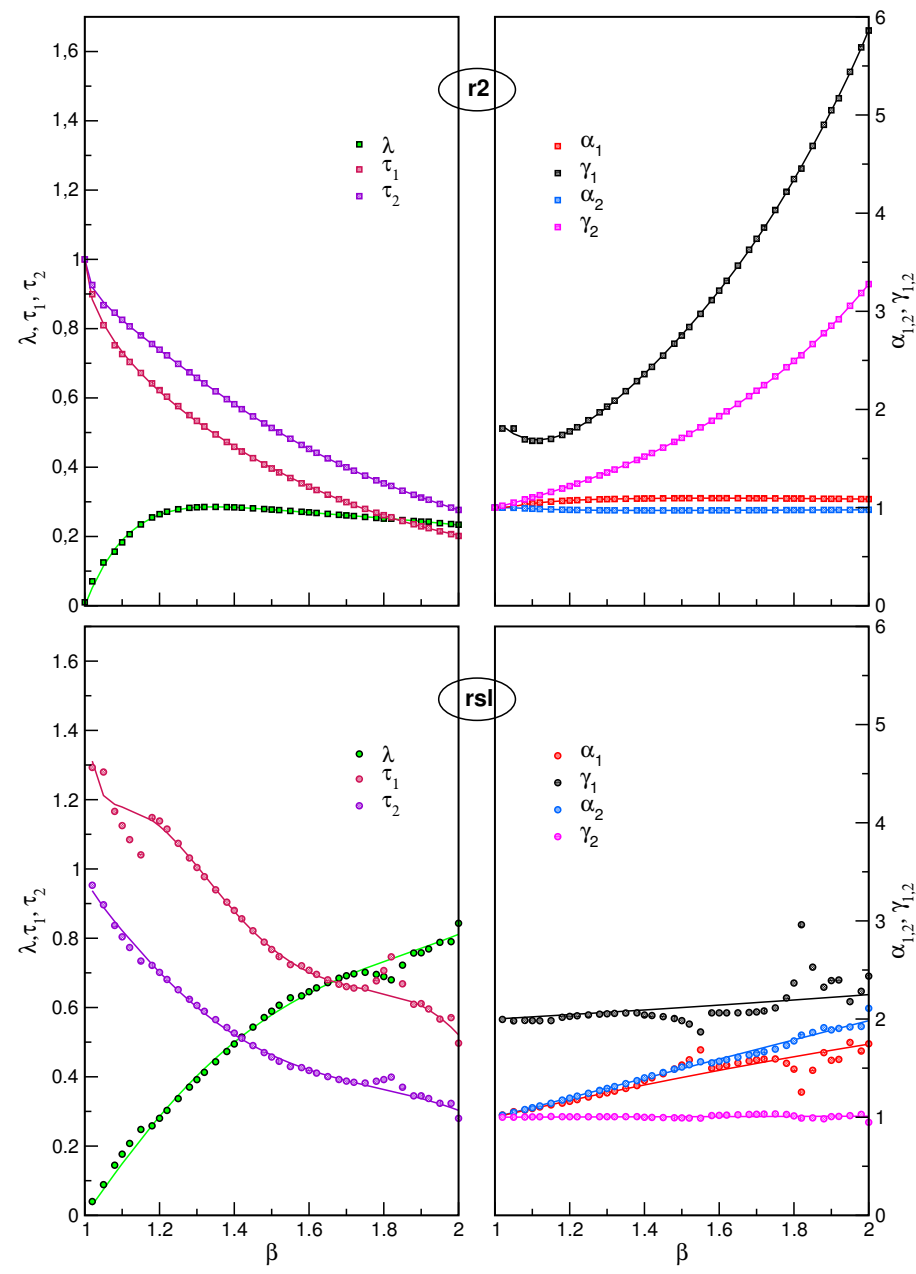

Figure 5. Same as Figure 3, in the interval of $1<\beta \leq$ 2. The corresponding mathematical expressions (see solid lines) appears in Tables A.5-A.8 in the appendix A. Note that for obtaining a smooth behavior and clear asymptotic trends, the following data points were omitted in the fitting procedure for the parameters given in Table A.5, A.7, and A.8: $\beta=1$ for $\gamma_{1}$ (see upper-right panel), $\beta=$ $1.50,1.52,1.55,1.80,1.82,1.85$ for $\alpha_{1}$ and $\gamma_{1}$ (see lower-right panel), and $\beta=1.12,1.15,1.78,1.80,1.82,1.85$ for $\tau_{1}, \tau_{2}$ and $\lambda$ (see lower-left panel) .

In Figure 6 the products $\alpha \cdot \gamma(\beta)$ for the sampling steps $\mathrm{r}_{1}, \mathrm{r}_{2}$ and $\mathrm{r}_{s l}$ are shown for the approximant $\mathcal{A} p_{1} H N$ in the interval of $0<\beta \leq 1$. One can see that $\alpha \cdot \gamma(r 1)$, follows a straight line $y=\beta$, with a regression to $y=\beta^{d}$ gives $d=1.035$. The second and third cases $\left(r_{2}\right.$ and $\left.r_{2 b}\right)$, give $d=1.143$ and $d=1.113$ respectively. In all three cases the dependency deviates from the empirical proposed law $y=\beta^{1.23}$ given in Ref. ([41, 42]).

As we mentioned previously, when we look at the interval of $1<\beta \leq 2$, the linear approach to $\alpha$. $\gamma(\beta)$ nevertheless fails, for same kind of $r_{1}, r_{2}$ and $r_{2 b}$

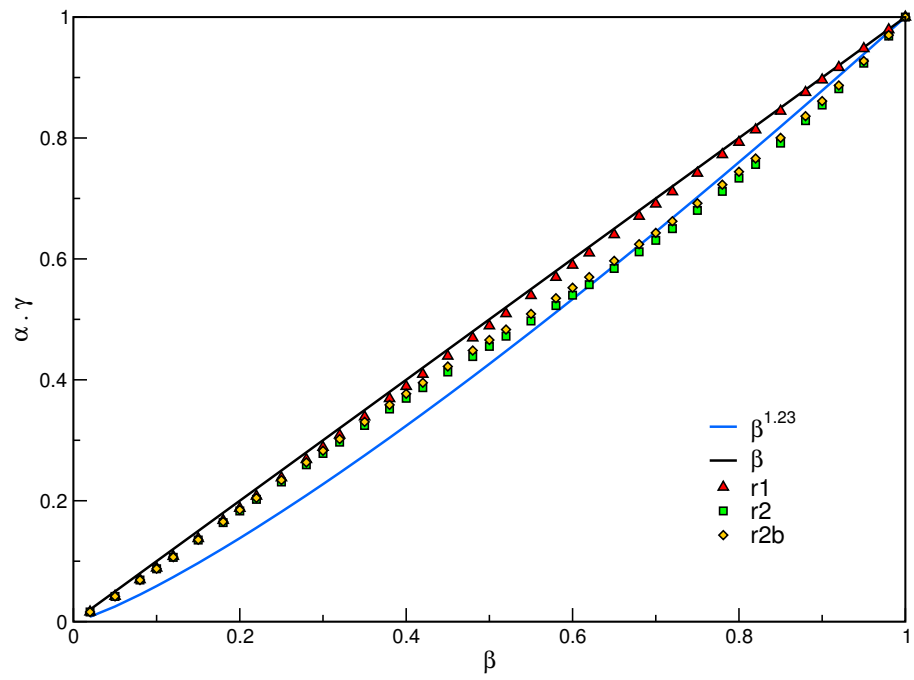

Figure 6. Asymptotic laws expressed as $\alpha \cdot \gamma$ for the approximant $\mathcal{A} p_{1} H N$ Eq. 1 , in the interval of $0<\beta \leq 1$, and using the sampling meshes $r_{1}$ (triangles up), $r_{2}$ (squares) and $r_{2 b}$ (diamonds). Solid lines corresponds to the situations $\alpha \cdot \gamma=\beta$ and $\alpha \cdot \gamma=\beta^{1.23}[41]$, respectively.

samplings. We should point out that, while adjusting $\psi_{\beta}$ to $\mathcal{A} p_{1} H N$, a progression from tail dominated, $(y \sim m * \beta, m<1)$, to body dominated fit, $\left(y \sim \beta^{a}, a>\right.$ 1 ), will take place in product $\alpha \cdot \gamma(\beta)$, only if a proper balance of weights, as well as finite windows effects, are taken into account. Then a unique $\mathrm{HN}$ function shall follow the asymptotic condition $\alpha \cdot \gamma=\beta^{d}$, with $d=1$. Otherwise, approximant $\mathcal{A} p_{1} H N$ won't work properly for the whole $0<\beta \leq 2$.

In Figure 7 all $\alpha \cdot \gamma$ are shown for the sampling steps $\mathrm{r}_{2}$ and $\mathrm{r}_{s l}$ when considering the approximant $\mathcal{A} p_{2} H N$ Eq. 2 , in the whole interval $0<\beta \leq 2$. Subscripts numbers 1 and 2, stand for the first (upper panels) and second (lower panels) $H N_{n}(\omega)$ terms in each approximant. For the case of $0<\beta \leq 1$, the asymptotic constraints for $\mathrm{r}_{s l}$ give $(\alpha \cdot \gamma)_{1,2}=\beta$, while for $\mathrm{r}_{2}$ the asymptotic law is $(\alpha \cdot \gamma)_{1,2} \sim \beta$. One can see how functional proximity can be improved by adding a new HN term [39, 19], compared with approximant $\mathcal{A} p_{1} H N$ (see Figure 6). Again, we observe how the expression of Alvarez et al [41] is a biased approximation for converting from time to frequency domain, or vice-versa. In the case of $1<\beta \leq 2$, the products $\alpha_{1} \cdot \gamma_{1}$ and $\alpha_{2} \cdot \gamma_{2}$ well behave, despite of their individual oscillations. One can see, that for high frequency or tail dominated values (the $\mathrm{r}_{s l}$ case) it's possible to use the $\mathcal{A} p_{2} H N$ approximant with 8 

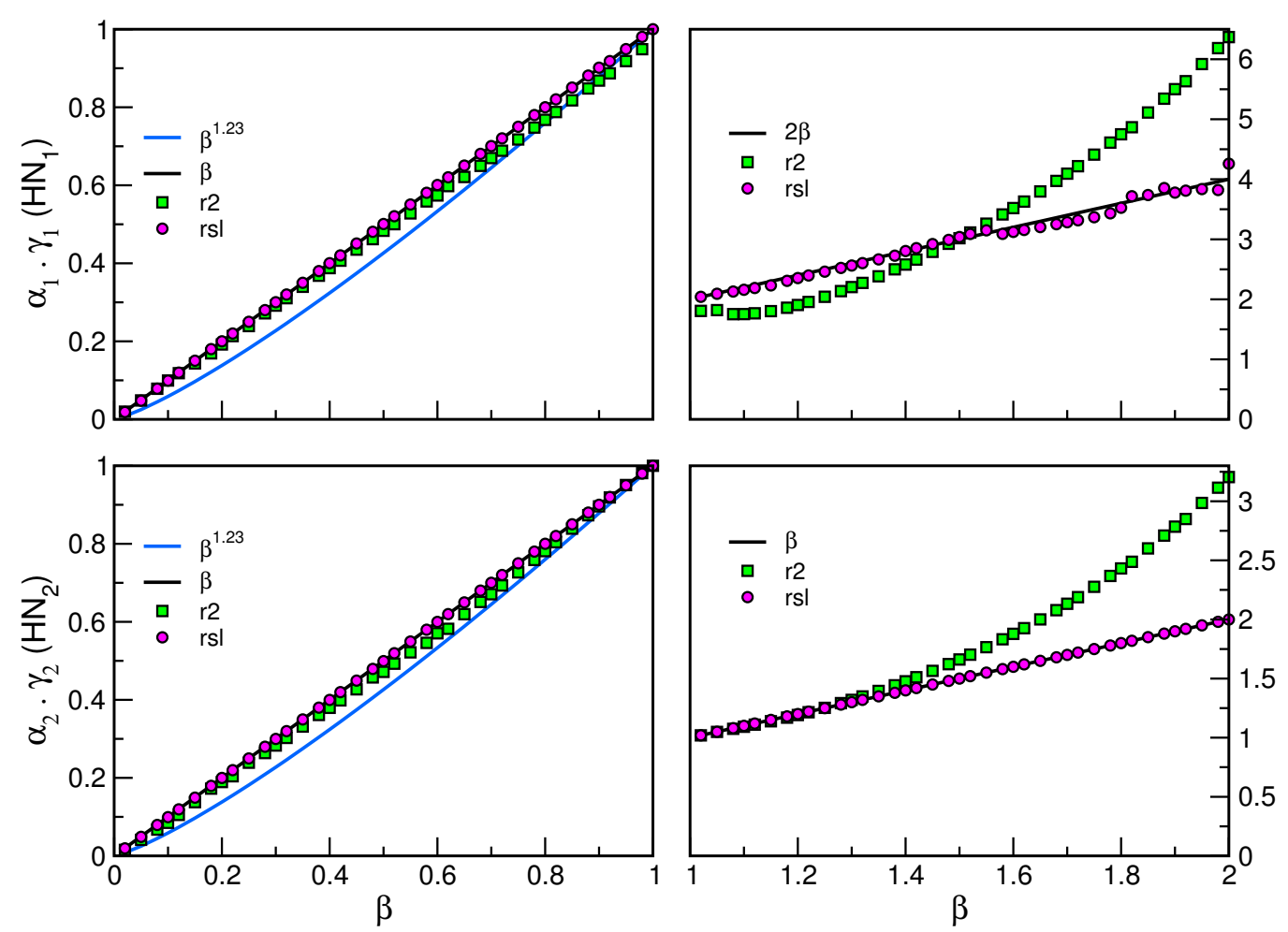

Figure 7. Asymptotic laws expressed as $\alpha_{1} \cdot \gamma_{1}$ (upper panels) and $\alpha_{2} \cdot \gamma_{2}$ (lower panels) for the approximant $\mathcal{A} p_{2} H N$ in the intervals of $0<\beta \leq 1$ (left side) and $1<\beta \leq 2$ (right side). The sampling meshes correspond to $r_{2}$ (squares) and $r_{s l}$ (circles). On the left side, solid lines represent the situations $\alpha \cdot \gamma=\beta$ and $\alpha \cdot \gamma=\beta^{1.23}$ [41], respectively. On the right side, the product $\alpha \cdot \gamma=\beta$ stands for the $1^{\text {st }} \mathrm{HN}$ term and $\alpha \cdot \gamma=2 \beta$ for the $2^{\text {nd }} \mathrm{HN}$ term.

However, when low frequencies or body dominates values are present (the $\mathrm{r}_{2}$ case) the constraints for $\alpha \cdot \gamma$ won't be fulfilled and the asymptotic laws should follow $(\alpha \cdot \gamma)_{1,2} \sim \beta^{d}$.

So far, we have analyzed the local asymptotic behavior of our approximants as a function of $\alpha$ and $\gamma$. This is more convenient regarding the use of $\tau_{1,2}$, since such parameters will depend heavily on powers of $\alpha_{1,2}$ and $\gamma_{1,2}$, thus the asymptotic constraints will not be enough to draw clear information if a good understanding of strengths and weaknesses of global model is absent. In this sense, our expressions can be employed for checking the consistency of models dealing with real data. This is a useful and quite unique tool, and in this sense, we put a step forward for building a general, coherent and compact model by providing a very exhaustive study of the parameter space of both approximants. Despite of choosing to present results on a general case without relying on the underlying physics, the ability or inability does not discount its use and further implications in other fields. However, these expressions will help to provide physical insights into the relax- ation phenomena and dynamical processes occurring on simple and complex fluids. Work in progress is oriented towards the global exploration of functional forms' sensibilities along the whole frequency range, as well as the $\beta$ parameter's relation with respect to thermodynamical conditions.

\section{Acknowledgments}

The authors thank to Centro de Calculo del IFF, SGAI (CSIC) and CESGA for allocation of computer time. This work has been supported by MINECO grant No. FIS2014-51933-P, and by COST Actions CM1204(X-LIC) and CM1405(MOLIM). DJAA acknowledges the FPI-MEC predoctoral fellowship BES-2012-054209.

\section{References}

[1] R. Kohlrausch, Theorie des elektrischen Rückstandes in der Leidener Flasche, Ann. Phys. 167 (1854) 56-82.

[2] R. Kohlrausch, Theorie des elektrischen Rückstandes in der Leidener Flasche, Ann. Phys. 167 (1854) 179-214. 
[3] G. Williams, D. C. Watts, Non-symmetrical dielectric relaxation behaviour arising from a simple empirical decay function, Trans. Faraday Soc. 66 (1970) 80-85.

[4] G. Williams, D. C. Watts, S. B. Dev, A. M. North, Further considerations of non symmetrical dielectric relaxation behaviour arising from a simple empirical decay function, Trans. Faraday Soc. 67 (1971) 1323-1335.

[5] C. P. Lindsey, G. D. Patterson, Detailed comparison of the Williams-Watts and ColeDavidson functions, J. Chem. Phys. 73 (1980) 3348-3357.

[6] P. Debye, Ver. Deut. Phys. Gesell. 15 (1913) 777, reprinted in collected papers of P.J.W. Debye, Interscience, New York (1954).

[7] K. S. Cole, R. H. Cole, Dispersion and absorption in dielectrics I. alternating current characteristics, J. Chem. Phys. 9 (1941) 341-351.

[8] D. W. Davidson, R. H. Cole, Dielectric relaxation in glycerol, propylene glycol, and npropanol, J. Chem. Phys. 19 (1951) 1484-1490.

[9] S. Havriliak, S. Negami, A complex plane analysis of $\alpha$ dispersions in some polymer systems, J. Polym. Sci. C: Polymer Symposia 14 (1966) 99-117.

[10] S. Havriliak, S. Negami, A complex plane representation of dielectric and mechanical relaxation processes in some polymers, Polymer 8 (1967) $161-210$.

[11] J.-U. Hagenah, G. Meier, G. Fytas, E. W. Fischer, Distribution of retardation times from the photon correlation spectra of glass forming systems, Polym. J. 19 (1987) 441-449.

[12] H. L. Lin, Y. S. Chen, T. L. Yu, Depolarized photon correlation spectroscopy and shear stress relaxation modulus of polystyrene, Polym. J. 26 (1994) 431-438.

[13] S. H. Chung, J. R. Stevens, Time-dependent correlation and the evaluation of the stretched exponential or Kohlrausch-Williams-Watts function, Am. J. Phys. 59 (1991) 1024-1030.

[14] K. B. Lee, J. Siegel, S. Webb, S. Lévêque-Fort, M. Cole, R. Jones, K. Dowling, M. Lever, P. French, Application of the stretched exponential function to fluorescence lifetime imaging, Biophys. J. 81 (2001) 1265 - 1274.

[15] G.-J. Guo, Y.-G. Zhang, K. Refson, Y. J. Zhao, Viscosity and stress autocorrelation function in supercooled water: a molecular dynamics study, Mol. Phys. 100 (2002) 26172627.

[16] M. Berberan-Santos, E. Bodunov, B. Valeur, Mathematical functions for the analysis of luminescence decays with underlying distributions 1 . Kohlrausch decay function (stretched exponential), Chem. Phys. 315 (2005) 171 -182 .

[17] G. Delgado-Barrio, R. Prosmiti, P. Villarreal, G. Winter, J. S. Medina, B. González, J. V. Alemán, J. L. Gomez, P. Sangrá, J. J. Santana, M. E. Torres, Viscosity of Liquid Water via Equilibrium Molecular Dynamics Simulations, Springer Netherlands, Dordrecht, 2008, pp. 351-361.

[18] J. S. Medina, R. Prosmiti, P. Villarreal, G. DelgadoBarrio, J. V. Alemán, B. González, G. Winter, Filtered stress autocorrelation functions of liquid water models, Inter. J. Quant. Chem. 111 (2011) 375-386.

[19] J. S. Medina, R. Prosmiti, P. Villarreal, G. DelgadoBarrio, J. V. Alemán, Frequency domain description of Kohlrausch response through a pair of Havriliak-Negamitype functions: An analysis of functional proximity, Phys. Rev. E 84 (2011) 066703.

[20] J. Medina, R. Prosmiti, P. Villarreal, G. Delgado-Barrio, G. Winter, B. González, J. Alemán, C. Collado, Molecular dynamics simulations of rigid and flexible water models: Temperature dependence of viscosity, Chem. Phys. 388 (2011) $9-18$.

[21] N. Ahmad, R. Adnan, J.-C. Soetens, C. Millot, Molecular dynamics simulations of liquid isoquinoline as a function of temperature, Chem. Phys. 407 (2012) 29 - 38.

[22] G. S. Fanourgakis, J. S. Medina, R. Prosmiti, Determining the bulk viscosity of rigid water models, J. Phys. Chem. A 116 (2012) 2564-2570.

[23] D. Arismendi-Arrieta, J. S. Medina, G. S. Fanourgakis, R. Prosmiti, G. Delgado-Barrio, Simulating liquid water for determining its structural and transport properties, Appl. Rad. Isot. 83 (2014) 115 - 121.

[24] Y. Zhang, A. Otani, E. J. Maginn, Reliable viscosity calculation from equilibrium molecular dynamics simulations: A time decomposition method, J. Chem. Theor. Comput. 11 (2015) 3537-3546.

[25] O. Russina, W. Schröer, A. Triolo, Mesoscopic structural and dynamic organization in ionic liquids, J. Mol. Liq. 210 (2015) $161-163$.

[26] A. Arbe, P. Malo de Molina, F. Alvarez, B. Frick, J. Colmenero, Dielectric Susceptibility of Liquid Water: Microscopic Insights from Coherent and Incoherent Neutron Scattering, Phys. Rev. Lett. 117 (2016) 185501-1-5.

[27] J. C. Phillips, Stretched exponential relaxation in molecular and electronic glasses, Rep. Prog. Phys. 59 (1996) 1133.

[28] A. A. Gurtovenko, Y. Y. Gotlib, Dynamics of inhomogeneous cross-linked polymers consisting of domains of different sizes, J. Chem. Phys. 115 (2001) 6785-6793.

[29] P. G. Debenedetti, F. H. Stillinger, Supercooled liquids and the glass transition, Nature 410 (2001) 259-267.

[30] B. Fierz, H. Satzger, C. Root, P. Gilch, W. Zinth, T. Kiefhaber, Loop formation in unfolded polypeptide chains on the picoseconds to microseconds time scale, PNAS 104 (2007) 2163-2168.

[31] A. R. Dobrovolskis, J. L. Alvarellos, J. J. Lissauer, Lifetimes of small bodies in planetocentric (or heliocentric) orbits, Icarus 188 (2007) $481-505$.

[32] S. Kuznetsova, G. Zauner, T. J. Aartsma, H. Engelkamp, N. Hatzakis, A. E. Rowan, R. J. M. Nolte, P. C. M. Christianen, G. W. Canters, The enzyme mechanism of nitrite reductase studied at single-molecule level, PNAS 105 (2008) 3250-3255.

[33] M. T. Viciosa, G. Pires, J. J. M. Moura Ramos, Is the Kohlrausch function a good tool to account for nonexponentiality in thermally stimulated depolarisation currents 
(TSDC) data treatment?, Chem. Phys. 359 (2009) 156 160.

[34] R. Kahlau, D. Kruk, T. Blochowicz, V. N. Novikov, E. A. Rössler, Generalization of the ColeDavidson and Kohlrausch functions to describe the primary response of glass-forming systems, J. Phys.: Condens. Matt. 22 (2010) 365101.

[35] K. Ngai, Relaxation and Diffusion in Complex Systems, Springer, 2011.

[36] S. A. Baeurle, A. Hotta, A. A. Gusev, A new semiphenomenological approach to predict the stress relaxation behavior of thermoplastic elastomers, Polymer 46 (2005) $4344-4354$.

[37] H. Scher, M. F. Shlesinger, J. T. Bendler, Timescale invariance in transport and relaxation, Phys. Today 44 (1991) 26-34.

[38] K. Weron, A. Jurlewicz, M. Patyk, A. Stanislavsky, The impact of hierarchically constrained dynamics with a finite mean of cluster sizes on relaxation properties, Ann. Phys. 332 (2012) 90 - 97.

[39] J. R. Macdonald, R. L. Hurt, Analysis of dielectric or conductive system frequency response data using the Williams-Watts function, J. Chem. Phys. 84 (1986) 496502.

[40] M. Schmollngruber, C. Schrder, O. Steinhauser, Polarization effects on the solvation dynamics of coumarin C153 in ionic liquids: Components and their cross-correlations, J. Chem. Phys. 138 (2013) 204504.

[41] F. Alvarez, A. Alegría, J. Colmenero, Relationship between the time-domain Kohlrausch-Williams-Watts and frequency-domain Havriliak-Negami relaxation functions, Phys. Rev. B 44 (1991) 7306-7312.

[42] F. Alvarez, A. Alegría, J. Colmenero, Interconnection between frequency-domain Havriliak-Negami and timedomain Kohlrausch-Williams-Watts relaxation functions, Phys. Rev. B 47 (1993) 125-130.

[43] S. Havriliak, S. Havriliak, Time to frequency domain transforms, Polymer 36 (1995) 2675 - 2680.

[44] H. Schäfer, E. Sternin, R. Stannarius, M. Arndt, F. Kremer, Novel approach to the analysis of broadband dielectric spectra, Phys. Rev. Lett. 76 (1996) 2177-2180.

[45] R. Díaz-Calleja, Comment on the maximum in the loss permittivity for the HavriliakNegami equation, Macromolecules 33 (2000) 8924-8924.

[46] G. H. Weiss, M. Dishon, A. M. Long, J. T. Bendler, A. A. Jones, P. T. Inglefield, A. Bandis, Improved computational methods for the calculation of KohlrauschWilliams/Watts (KWW) decay functions, Polymer 35 (1994) 1880 - 1883.

[47] R. Ferguson, V. Arrighi, I. J. McEwen, S. Gagliardi, A. Triolo, An improved algorithm for the Fourier integral of the KWW function and its application to neutron scattering and dielectric data, J. Macromolec. Sci. B 45 (2006) 1065-1081.

[48] J. Wuttke, Laplace-Fourier transform of the stretched exponential function: Analytic error bounds, double ex- ponential transform, and open-source implementation libkww, Algorithms 5 (2012) 604628.

[49] C. R. Snyder, F. I. Mopsik, Critical comparison between time- and frequency-domain relaxation functions, Phys. Rev. B 60 (1999) 984-990.

[50] E. Helfand, On inversion of the WilliamsWatts function for large relaxation times, J. Chem. Phys. 78 (1983) 19311934.

[51] J. H. Wu, Q. Jia, The heterogeneous energy landscape expression of KWW relaxation, Scient. Rep. 6 (2016) 20506.

[52] E. Cuervo-Reyes, Why the dipolar response in dielectrics and spin-glasses is unavoidably universal, Scient. Rep. 6 (2016) 29021.

[53] W. Weibull, A statistical distribution function of wide applicability, J. Appl. Mechan. 18 (1951) 293-297.

[54] H. Rinne, The Weibull Distribution A Handbook, CRC Press, Taylor \& Francis Group, 2008.

[55] S. Havriliak, Jr, S.J. Havriliak, Comparison of the Havriliak-Negami and stretched exponential functions, Polymer 37 (1996) 4107 - 4110.

[56] W. Research, Mathematica 8.0 (2010).

[57] P. Turner, E. Stambulchik, G. D. Team, see http://plasmagate.weizmann.ac.il/Grace/.

\section{Appendix A. $\{\alpha, \gamma, \tau, \lambda\}(\beta)$ Relationships}

Below, we provide the mathematical expressions that describe the curves from Figures 3 and 5 as a function of $\beta$, for both the stretched $(\beta \leq 1)$ and squezeed $(\beta>1)$ instances. Each of the expressions were designed to match the parameters curves with a non-linear fitting procedure [57]. Corr. stands for the corresponding correlation coefficient for each fit. 


\begin{tabular}{|c|c|c|c|c|c|c|}
\hline \multirow[t]{2}{*}{ Table A.1 } & \multicolumn{3}{|c|}{$\beta A \exp \left[\left(\sum_{s=1}^{3} a_{s}(1-\beta)^{s}\right) \exp \left(-M \beta^{d}\right)\right]$} & \multicolumn{3}{|c|}{1} \\
\hline & & $\alpha_{1}$ & $\alpha_{2}$ & & $\gamma_{1}$ & $\gamma_{2}$ \\
\hline \multirow{7}{*}{$0<\beta \leq 1\left(\mathrm{r}_{s l}\right)$} & $A$ & 1.68125 & 1 & $A$ & 1.6738 & 1.000 \\
\hline & $M$ & 6.00482 & 2.14961 & $M$ & 4.95993 & 2.49211 \\
\hline & $d$ & 0.712224 & 0.580585 & $d$ & 1.17881 & 0.651276 \\
\hline & $a_{1}$ & -30.8645 & 0.56705 & $a_{1}$ & -5.87091 & 0.585986 \\
\hline & $a_{2}$ & 75.8761 & -2.83736 & $a_{2}$ & 13.4251 & -2.92872 \\
\hline & $a_{3}$ & -44.5841 & 2.97408 & $a_{3}$ & -6.94891 & 3.0715 \\
\hline & Corr. & 0.999858 & 0.999997 & Corr. & 0.997978 & 0.999256 \\
\hline
\end{tabular}

\begin{tabular}{|c|c|c|c|c|c|c|}
\hline Table A.2 & \multicolumn{2}{|c|}{$\begin{aligned} \Sigma_{s=1}^{2} & \left\{A_{s}(1-\beta)+B_{s}(1-\beta)^{2}\right\} \\
& \exp \left(-M_{s} \beta^{0.2}\right)+C_{c}\end{aligned}$} & \multicolumn{2}{|c|}{$\beta^{3}+\beta^{d} \sum_{s=1}^{7} b_{s}(1-\beta)^{s}$} & \multicolumn{2}{|c|}{$\exp \left(-M \beta^{d}\right) \sum_{s=1}^{5} c_{s}(1-\beta)^{s}$} \\
\hline \multirow{10}{*}{$0<\beta \leq 1\left(\mathrm{r}_{s l}\right)$} & & $\log _{10} \tau_{1}$ & & $\tau_{2}$ & & $\lambda$ \\
\hline & $\overline{A_{1}}$ & -22.4446 & $d$ & 2.96596 & $M$ & 4.14933 \\
\hline & $B_{1}$ & -6.64276 & $b_{1}$ & 4.54749 & $d$ & 3.1579 \\
\hline & $M_{1}$ & -0.233028 & $b_{2}$ & -32.4329 & $c_{1}$ & 3.43714 \\
\hline & $A_{2}$ & 8231.54 & $b_{3}$ & 552.577 & $c_{2}$ & -12.6851 \\
\hline & $B_{2}$ & -7799.77 & $b_{4}$ & -3159.47 & $c_{3}$ & 35.8224 \\
\hline & $M_{2}$ & 5.59666 & $b_{5}$ & 9513.52 & $c_{4}$ & -46.5211 \\
\hline & $C_{c}$ & -0.65664 & $b_{6}$ & -13492.1 & $c_{5}$ & 20.5172 \\
\hline & & & $b_{7}$ & 7139.92 & & \\
\hline & Corr. & 0.999984 & Corr. & 0.998613 & Corr. & 0.998905 \\
\hline
\end{tabular}

\begin{tabular}{|c|c|c|c|c|c|c|}
\hline \multirow[t]{2}{*}{ Table A.3 } & \multicolumn{3}{|c|}{$\beta \exp \left[\left(\sum_{s=1}^{3} a_{s}(1-\beta)^{s}\right) \exp \left(M \beta^{d}\right)\right]$} & \multicolumn{3}{|c|}{$\frac{A}{\exp \left[\left(\Gamma^{3} \quad a_{s}(1-\beta)^{s}\right) \exp \left(M \beta^{d}\right)\right]}$} \\
\hline & & $\alpha_{1}$ & $\alpha_{2}$ & & $\gamma_{1}$ & $\gamma_{2}$ \\
\hline \multirow{7}{*}{$0<\beta \leq 1\left(\mathrm{r}_{2}\right)$} & $M$ & 3.6903 & 2.42224 & $M$ & 4.54457 & 3.4752 \\
\hline & $d$ & 1.4618 & 1.03791 & $d$ & 1.62482 & 1.47877 \\
\hline & $a_{1}$ & 0.0190435 & 0.0962135 & $A$ & 0.999202 & 1.00287 \\
\hline & $a_{2}$ & 0.0794805 & 0.193794 & $a_{1}$ & 0.0227205 & 0.0401981 \\
\hline & $a_{3}$ & 0.572997 & 0.761107 & $a_{2}$ & -0.055399 & 0.054449 \\
\hline & & & & $a_{3}$ & 0.79403 & 1.29633 \\
\hline & Corr. & 0.999950 & 0.999942 & Corr. & 0.998473 & 0.999654 \\
\hline
\end{tabular}

\begin{tabular}{|c|c|c|c|c|c|c|}
\hline \multirow[t]{2}{*}{ Table A.4 } & \multicolumn{2}{|c|}{$\beta^{d} \sum_{s=0}^{4} b_{s}(1-\beta)^{s}$} & \multicolumn{2}{|c|}{$\begin{array}{c}\sum_{s=1}^{2}\left\{A_{s}(1-\beta)+B_{s}(1-\beta)^{2}\right\} \\
\exp \left(-M_{s} \beta^{0.2}\right)\end{array}$} & \multicolumn{2}{|c|}{$\exp [-M(1-\beta)] \sum_{s=1}^{5} c_{s}(1-\beta)^{s}$} \\
\hline & & $\tau_{1}$ & & $\log _{10} \tau_{2}$ & & $\lambda$ \\
\hline \multirow{7}{*}{$0<\beta \leq 1\left(\mathrm{r}_{2}\right)$} & $d$ & 0.785731 & $A_{1}$ & 42.4745 & $M$ & 4.60773 \\
\hline & $b_{0}$ & 0.726519 & $B_{1}$ & -49.3268 & $c_{1}$ & 4.80377 \\
\hline & $b_{1}$ & 0.160075 & $M_{1}$ & 3.65441 & $c_{2}$ & -12.6617 \\
\hline & $b_{2}$ & 7.16583 & $A_{2}$ & 66182.6 & $c_{3}$ & 78.3249 \\
\hline & $b_{3}$ & -14.5815 & $B_{2}$ & -61430.8 & $c_{4}$ & -120.494 \\
\hline & $b_{4}$ & 8.47342 & $M_{2}$ & 12.7917 & $c_{5}$ & 89.8475 \\
\hline & Corr. & 0.997479 & Corr. & 0.999987 & Corr. & 0.996847 \\
\hline
\end{tabular}




\begin{tabular}{|c|c|c|c|c|c|c|}
\hline Table A.5 & \multicolumn{3}{|c|}{$1+\exp \left(\frac{-M}{\beta-1+\epsilon}\right) \sum_{s=0}^{3} a_{s}(\beta-1)^{s}$} & \multicolumn{3}{|c|}{$A+\exp \left(M(\beta-1)^{3}\right) \sum_{s=1}^{4} b_{s}(\beta-1)^{s}$} \\
\hline \multirow{8}{*}{$1<\beta \leq 2\left(\mathrm{r}_{2}\right)$} & & $\alpha_{1}$ & $\alpha_{2}$ & & $\gamma_{1}$ & $\gamma_{2}$ \\
\hline & $M$ & 0.116811 & 0.165105 & $A$ & 1.90381 & $\equiv 1.000$ \\
\hline & $a_{0}$ & 0.136577 & -0.0645705 & $M$ & 2.25005 & 0.239754 \\
\hline & $a_{1}$ & -0.0269458 & 0.0697225 & $b_{1}$ & -4.09964 & 0.948757 \\
\hline & $a_{2}$ & -0.00692586 & -0.0485374 & $b_{2}$ & 22.844 & 0.634155 \\
\hline & $a_{3}$ & -0.00572689 & 0.0171122 & $b_{3}$ & -29.9149 & 0.692054 \\
\hline & $\epsilon$ & \multicolumn{2}{|c|}{$10^{-180}$} & $b_{4}$ & 11.5881 & -0.484575 \\
\hline & Corr. & 0.999249 & 0.999926 & Corr. & 0.999956 & 0.999993 \\
\hline
\end{tabular}

\begin{tabular}{|c|c|c|c|c|c|}
\hline Table A.6 & \multicolumn{3}{|c|}{$c_{0} \sqrt{(\beta-1)}+\sum_{s=1}^{4} c_{s}(\beta-1)^{s}$} & \multicolumn{2}{|c|}{$\exp [-M(\beta-1)] \sum_{s=1}^{3} d_{s}(\beta-1)$} \\
\hline \multirow{7}{*}{$1<\beta \leq 2\left(\mathrm{r}_{2}\right)$} & & $-\log _{10} \tau_{1}$ & $-\log _{10} \tau_{2}$ & & $\lambda$ \\
\hline & $c_{0}$ & 0.339472 & 0.27042 & $M$ & 2.9177 \\
\hline & $c_{1}$ & 0.27034 & -0.1091 & $d_{1}$ & 2.80567 \\
\hline & $c_{2}$ & 0.0194173 & 0.977286 & $d_{2}$ & -3.18276 \\
\hline & $c_{3}$ & 0.26079 & -0.884491 & $d_{3}$ & 4.6885 \\
\hline & $c_{4}$ & -0.198826 & 0.303991 & & \\
\hline & Corr. & 0.999853 & 0.999961 & Corr. & 0.998497 \\
\hline
\end{tabular}

\begin{tabular}{|c|c|c|c|c|c|c|}
\hline Table A.7 & \multicolumn{3}{|c|}{$\beta\left(1+a_{1}(\beta-1)\right)$} & \multicolumn{3}{|c|}{$\frac{A}{\left(1+a_{1}(\beta-1)\right)}$} \\
\hline \multirow{4}{*}{$1<\beta \leq 2\left(\mathrm{r}_{s l}\right)$} & & $\alpha_{1}$ & $\alpha_{2}$ & & $\gamma_{1}$ & $\gamma_{2}$ \\
\hline & $a_{1}$ & -0.127772 & -0.00819886 & $A$ & 2 & 1 \\
\hline & & & & $a_{1}$ & -0.110735 & -0.0102285 \\
\hline & Corr. & 0.981962 & 0.994925 & Corr. & 0.833974 & $0.096606 !$ \\
\hline
\end{tabular}

\begin{tabular}{|c|c|c|c|c|c|}
\hline Table A.8 & \multicolumn{3}{|c|}{$B+b_{0} \sqrt{\beta-1}+\sum_{s=1}^{4} b_{s}(\beta-1)^{s}$} & \multicolumn{2}{|c|}{$\exp [-M \sqrt{\beta-1}] \sum_{s=1}^{3} c_{s}(\beta-1)^{s}$} \\
\hline \multirow{8}{*}{$1<\beta \leq 2\left(\mathrm{r}_{s l}\right)$} & & $-\log _{10} \tau_{1}$ & $-\log _{10} \tau_{2}$ & & $\lambda$ \\
\hline & $A$ & -0.278737 & 0 & $M$ & -0.504694 \\
\hline & $b_{0}$ & 1.69465 & 0.165075 & $c_{1}$ & 1.41581 \\
\hline & $b_{1}$ & -4.114 & 0.219429 & $c_{2}$ & -1.49885 \\
\hline & $b_{2}$ & 9.33908 & 1.33322 & $c_{3}$ & 0.572827 \\
\hline & $b_{3}$ & -10.9942 & -2.39982 & & \\
\hline & $b_{4}$ & 4.63626 & 1.20034 & & \\
\hline & Corr. & 0.996880 & 0.999016 & Corr. & 0.998585 \\
\hline
\end{tabular}

